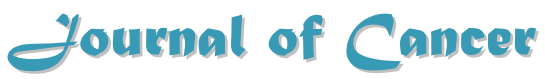

2010; 1:93-97

Research Paper

(C) Ivyspring International Publisher. All rights reserved

\title{
Dual use of a single Wilms' tumor 1 immunohistochemistry in evaluation of ovarian tumors: a preliminary study of 20 cases
}

\author{
Yi-Hsuan Hsiao ${ }^{1,2}$, Sarwat Siddiquiं ${ }^{3}$, Yan-gao Man ${ }^{凶}$ \\ 1. Institute of Medicine, Chung Shan Medical University, Taiwan \\ 2. Department of Obstetrics and Gynecology, Changhua Christian Hospital, Taiwan \\ 3. Department of Pathology, Howard University Hospital, Washington, DC \\ 4. Department of Gynecologic and Breast Pathology, Armed Forces Institute of Pathology and American Registry of Pa- \\ thology, Washington DC and Jilin University, Changchun, Jilin, China
}

Corresponding author: Yan-gao Man, MD., PhD., Director of Gynecologic and Breast Research Laboratory, Department of Gynecologic and Breast Pathology, Armed Forces Institute of Pathology and American Registry of Pathology. Tel: 202-782-1612; Fax: 202-782-3939; E-mail: man@afip.osd.mil

Published: 2010.07.13

\begin{abstract}
Our previous studies revealed that a single Wilms' tumor I (WT-I) immunohistochemistry can be used to elucidate both the myoepithelial cells and blood vessels of human breast tumors. As the human ovary is rich in blood vessels, and WT-I has been used as a biomarker for ovarian tumors, our current study attempted to assess if a single WT-I immunohistochemistry has dual usages in evaluation of ovarian tumor and endothelial cells. Paraffin-embedded tissue sections were prepared from 20 cases of ovarian tumors. A set of four consecutive sections from each case were subjected to immunohistochemistry with a mouse monoclonal antibody against the human WT-I protein, a well defined ovarian tumor marker, CA-125, and a endothelial cell phenotypic marker, CD34, respectively. From each case, 4-5 randomly selected fields were photographed, and expression of these molecules in the same structures were compared. Distinct WT-I immunoreactivities were seen in both ovarian tumor and endothelial cells. Over $90 \%$ of WT-I positive tumor and endothelial cells were positive for CA-I 25 and CD34, respectively. Similarly, over $90 \%$ of CA- 125 or CD34 positive cells co-express WT-I in tumor or endothelial cells, respectively. Our findings suggest that a single WT-I immunohistochemistry can be used to assess both the tumor cells and micro-vascular density in ovarian tumors. Our findings also suggest that as WT-I is expressed in both tumor and endothelial cells, the development of therapeutic agents to target WT-I may provide an effective treatment option for ovarian cancer.
\end{abstract}

Key words: Cancer biomarkers; WT-1 protein; vascular density; tumor invasion; ovarian tumors

\section{Introduction}

The Wilms' tumor 1 (WT-1) gene is located at chromosome 11p13, and is encoded by 10 exons, resulting in a WT-1 mRNA with a complex pattern of alternative splicing [1-5]. The WT-1 encodes a transcription factor of the zinc-finger family, which binds to GC-rich sequences and regulates the expression of several genes of the growth factor family, including the insulin-like growth factor and transforming growth factor [1-5]. The WT-1 protein is preferentially expressed in the genitourinary system and aberrant expression of the WT-1 protein has been implicated in the development of Wilms' tumors in this system [1-5]. Recent studies have further shown that aberrant expression of the WT-1 protein may also be closely 
associated with the development and progression of other malignancies, including mesothelioma, leukemia, and breast, esophageal, colorectal tumors [6-11]. Our own studies have consistently demonstrated that the WT-1 protein is preferentially present in breast myoepithelial and endothelial cells, and have shown that a single WT-1 immunohistochemistry can have dual use in evaluation of breast tumors [12,13].

As the human ovary is rich in blood vessels and WT-1 has been used as a biomarker for ovarian tumors [14-16], our current study attempted to test a hypothesis that WT-1 is co-expressed with a well defined ovarian tumor marker, CA125 [17-19], and also with a endothelial cell phenotypic marker, CD34, in the same cells. Therefore, a single WT-1 immunohistochemistry may have dual usages in evaluation of both the ovarian tumor cells and also the vascular density of the ovarian tissues.

\section{Materials and Methods}

Formalin-fixed, paraffin-embedded ovarian tissue blocks from 20-patients were retrieved from the files of the Armed Forces Institute of Pathology. Each of the tumors harbored normal and malignant tissue components. As the sole purpose of this study was to assess the overall expression of WT-1 in tumor and endothelial ells, the clinical profile was not collected and evaluated.

Consecutive sections at 4-5 $\mu \mathrm{m}$ thickness were cut and placed on positively charged slides. A set of four consecutive sections from each case were subjected to immunohistochemistry with mouse monoclonal antibodies against the human WT-1 protein (6F-H2; Cell Marque, Hot Springs, AR), the ovarian specific antigen CA-125 (Ov185:1; Lab Vision, Fremont, CA), the WT-1, and an endothelial cell marker, CD 34 (Vector, Burlingame, CA), according to the protocols provided by manufacturers. From each case, 4-5 fields with tumor cells were randomly selected and photographed, and enlarged prints were made. The numbers of positive and negative tumor or endothelial cells within the same structure (a tumor nest or blood vessel) were counted and the percentage of positive cells was calculated. Co-expression of WT-1 with CA-125 or CD34 was evaluated in two ways: (1) 2-3 prints with distinct WT-1 positive immunostaining were first selected from each case and used as positive controls to compare the expression of CA-125 and CD34 in adjacent sections, and (2) 1-2 prints with distinct CA-125 or CD34 immunostaining were first identified from each case and used as positive controls to compare the expression of WT- 1 in adjacent sections. Co-expression of WT- 1 with CA-125 or CD34 was defined as the presence of these molecules within identical cells at the adjacent sections.

To assess the specificity of the immunostaining, different controls were used, which included: (1) the substitution of the primary antibody with the normal serum, (2) the omission of the secondary antibody, (3) and serial dilutions of the primary antibody. All the immunostainings was repeated at least twice under the same condition. Immunostained sections were independently evaluated by investigators. A cell was considered positive if distinct immunoreactivities were consistently present in its cytoplasm or nucleus in all the repeated staining processes, whereas all negative controls were completely devoid of immunoreactivities.

\section{Results}

All negative controls were completely devoid of distinct immunoreactivities to WT-1, CA-125, and CD34. Distinct nuclear WT-1 expression was exclusively or preferentially seen in serous tumor cells, while both nuclear and/or cytoplasmic WT-1 expression was present in morphologically distinct endothelial cells in each of the cases, with weak and diffuse staining occasionally seen in some fibroblasts and white blood cells. CA-125 immunoreactivities were exclusively or preferentially seen in the surface plasma membrane of tumor cells. CD34 expression is seen mainly in the cytoplasm of endothelial cells.

The overall expression pattern and frequency of WT-1 and CA-125, or WT-1 and CD34, were very similar in all 94-randomly selected fields. Over 3,000 WT-1 positive tumor cells counted, $90.4 \%$ showed distinct CA-125 expression in the adjacent section (Fig 1). Over 3,000 CA-125 positive tumor cells counted, 90.3\% showed distinct WT-1 expression in the adjacent section (Fig 2). Similarly, over $90 \%$ of the WT-1 or CD34 positive endothelial cells showed distinct expression of their counterparts in the adjacent sections (Figs 1-2). 


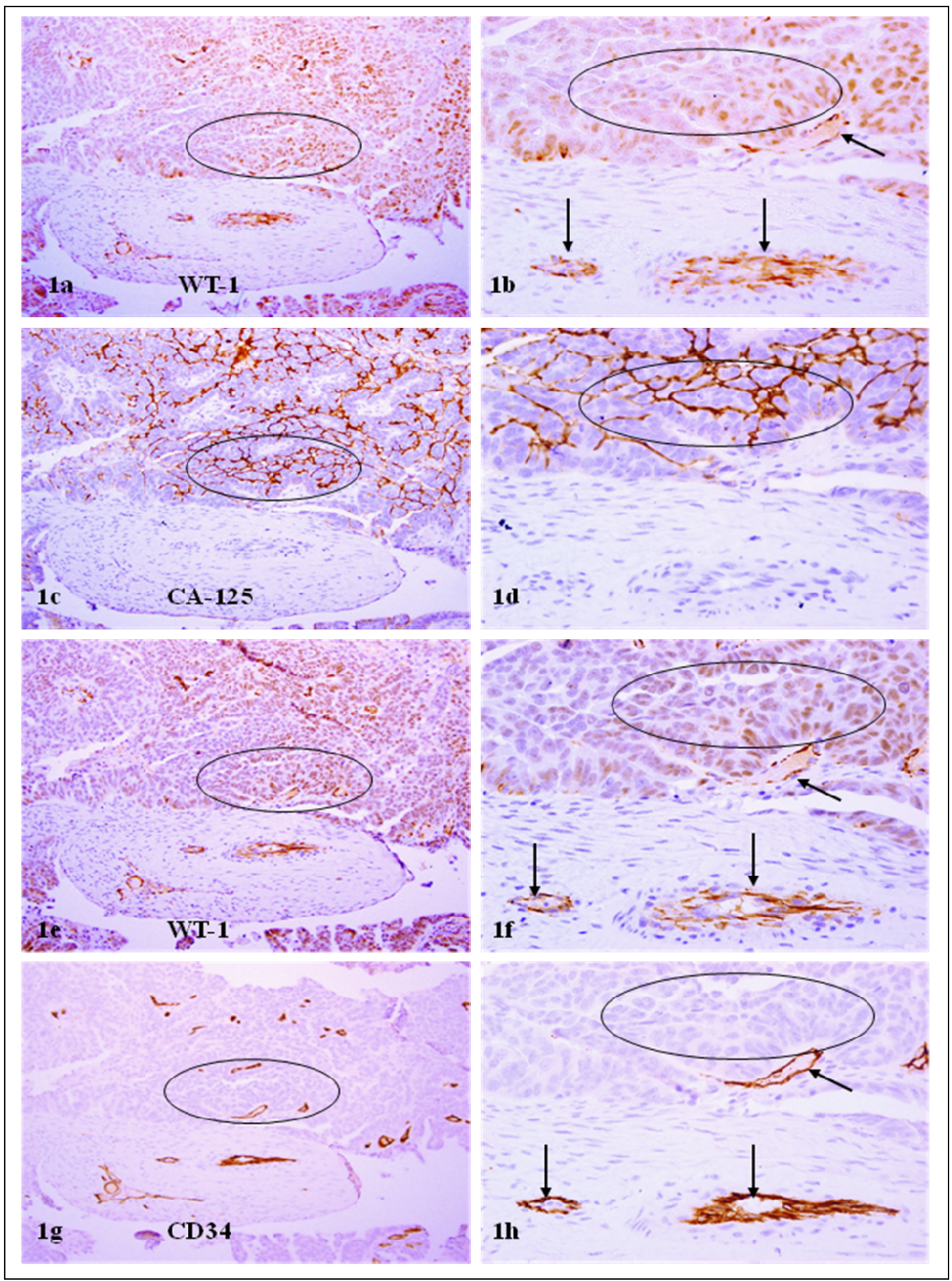

Figure I: WT-I positive cells co-express CA-I25 and CD34. A set of 4-consecutive sections were immunostained for WT-I, CA-I25, and CD34. Circles identify tumor cell clusters with WT-I and CA-I25 expression. Arrows identify small blood vessels. Note that a vast majority of WT-I positive cells co-express CA-I 25 or CD34, and that WT-I expression was consistent in different sections. a, c, e, and g: 100X. b, d, f, and h: a higher (300X) magnification of a, c, e, and g, respectively. 


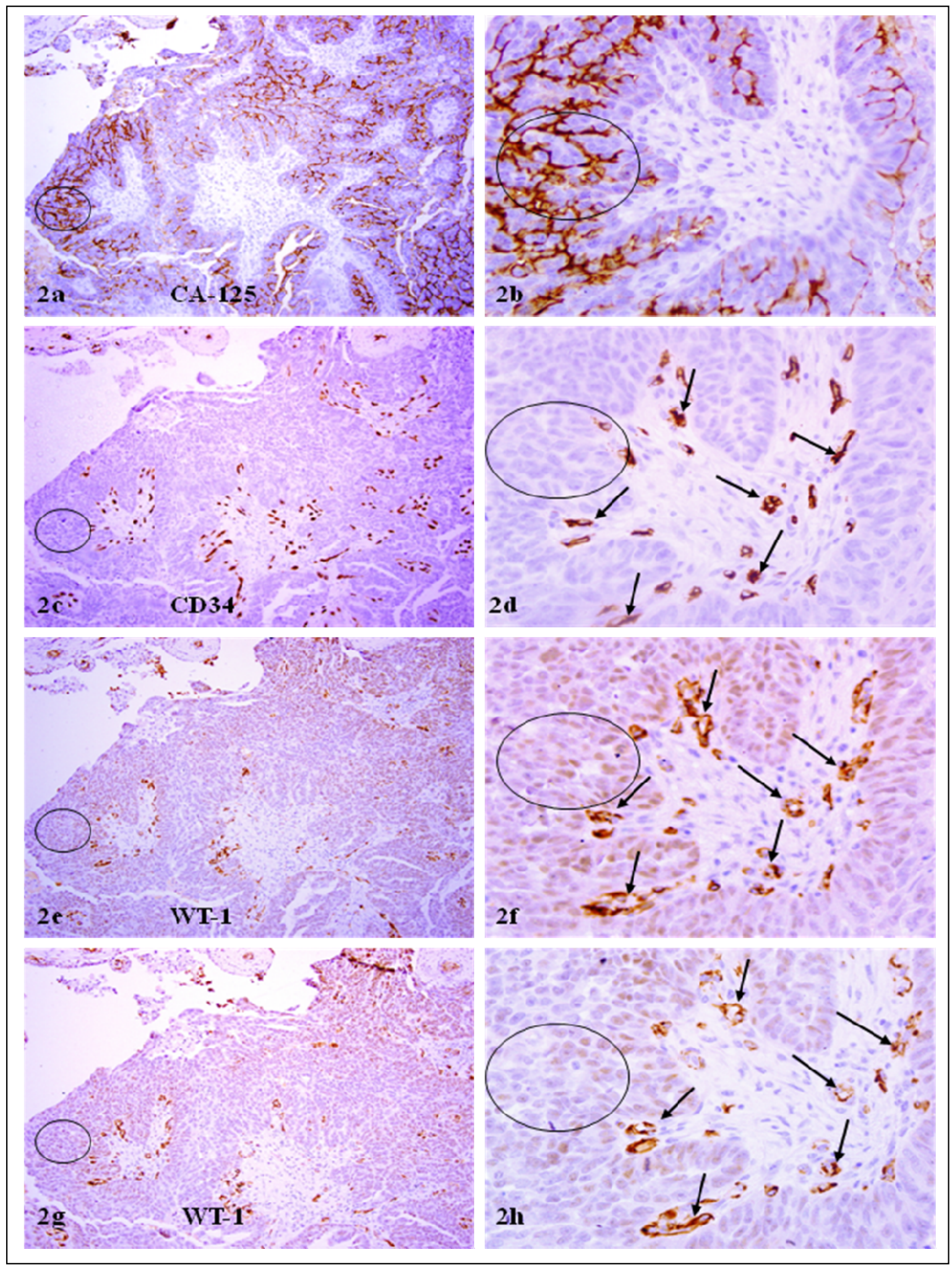

Figure 2: CA-I 25 or CD 34 positive cells co-express WT-I. A set of 4-consecutive sections were immunostained for CA-I25, CD34, and WT-I. Circles identify tumor cell clusters with WT-I and CA-I 25 expression. Arrows identify small blood vessels. Note that a vast majority of CA-I25 or CD34 positive tumor or endothelial cells co-express WT-I, and that WT-I expression was consistent in different sections. a, c, e, and g: 100X. b, d, f, and h: a higher (300X) magnification of a, c, e, and g, respectively. 


\section{Discussion and Conclusions}

Our current study revealed that: (1) over $90 \%$ of WT-1 positive tumor cells co-expressed CA-125, (2) over $90 \%$ of WT-1 positive endothelial cells co-expressed CD34, and (3) over $90 \%$ of the CA-125 or CD34 positive cells co-expressed their counterpart molecule WT-1 in tumor and endothelial cells, respectively. Our finding of WT-1 expression in ovarian tumor cells is consistent with a number of previous studies, which have shown that WT-1 is a useful marker for elucidation of ovarian tumor cells [14-16]. Our finding of WT-1 expression in endothelial cells is in total agreement with that of our previous study in human breast tumors [13] and is also consistent with that of a recent study, which reported that "Endothelial WT-1 expression was detected in 95\% of 113 tumors of different origin" [20]. Together, these findings suggest that a single WT-1 immunohistochemistry have dual usages in evaluation of both ovarian tumor cells and the vascular density. These findings also suggest that as WT-1 is heavily expressed in both tumor and endothelial cells, the development of therapeutic agents to specifically target WT- 1 may provide an effective treatment option for ovarian cancer.

\section{Acknowledgment}

This study was supported in part by grants DAMD17-01-1-0129, DAMD17-01-1-0130, PC051308 from Congressionally Directed Medical Research Programs, BCTR0706983 from The Susan G. Komen Breast Cancer Foundation, 05AA from AFIP/ARP joint research initiative projects to Dr. Yan-gao Man, and 2006CB910505 from the Ministry of Chinese Science and Technology Department.

The opinions and assertions contained herein represent the personal views of the authors and are not to be construed as official or as representing the views of the Department of the Army or the Department of Defense.

\section{Conflict of Interest}

The authors have declared that no conflict of interest exists.

\section{References}

1. Scharnhorst V, van der Eb AJ, Jochemsen AG. WT1 proteins: functions in growth and differentiation. Gene 2001; 273:141-161.

2. Re GG, Hazen-Martin DJ, Sens DA, Garvin AJ. Nephroblastoma (Wilms' tumor): a model system of aberrant renal development. Semin Diagn Pathol 1994; 11: 126-135.

3. Lee SB, Harber DA. Wilmas' tumor and WT1 gene. Exp Cell Res 2001; 264:74-99.
4. Hirose M. The role of Wilms' tumor genes. J Med Invest 1999; 46:130-140.

5. Scharnhorst V, Menke AL, Attema J, Haneveld JK, Riteco N, van Steenbrugge GL, et al. EGR-1 enhances tumor growth and modulates the effect of the Wilms' tumor 1 gene products on tumorgenicity. Oncogene 2000; 19:791-800.

6. Pu PI, Pang Y, Michael CW. Utility of WT-1, p63, MOC31, mesothelin, and cytokeratin (K903 and CK5/6 immunostainins in differentiating adenocarcinoma, squamous cell carcinoma, and malignant mesothelioma in effusions. Diagn Cytopathol 2008; 36: 20-25.

7. Yang L, Han Y, Suarez Saiz F, Minden MD. A tumor suppressor and oncogene: the WT-1 story. Leukemia 2007; 21: 868-876.

8. Caldon CE, Lee CS, Sutherland RL, Musgrove EA. Wilms' tumor protein 1: an early target of progestin regulation in T-47D breast cancer cells that modulates proliferation and differentiation. Oncogene 2008; 27: 126-138.

9. Fabre A, McCabb AH, O'Shea D, Broderick D, Keating G, Tobin $\mathrm{B}$, et al. Loss of heterozygosity of the Wilms' tumor suppressor gene (WT-1) in in-situ and invasive breast carcinoma. Hum Pathol 1999; 30: 661-665.

10. Oji Y, Yano M, Nakano Y, Abeno S, Nakatsuka S, Ikeba A, et al. Overexpression of the Wilms' tumor gene WT1 in esophageal cancer. Anticancer Res 2004; 24: 3103-3108.

11. Oji Y, Yamamoto H, Nomura M, Nakano Y, Ikeba A, Nakatsuka $\mathrm{S}$, et al. Overexression of the Wilms tumor gene WT1 in colorectal adenocarcinoma. Cancer Sci 2003; 94: 712-717.

12. Man YG, Sang QXA. The significance of focal myoepitehlial cell layer disruptions in breast tumor invasion: a paradigm shift from the "protease-centered" hypothesis. Exp Cell Res 2004; 301:103-118.

13. Li JH, Man YG. Dual usages of single wilms' tumor 1 immunohistochemistry in evaluation of breast tumors: a preliminary study of 30 cases. Cancer Biomarkers 2009; 5: 109-116.

14. Köbel M, Kalloger SE, Carrick J, Huntsman D, Asad H, Oliva E, Ewanowich CA, Soslow RA, Gilks CB. A limited panel of immunomarkers can reliably distinguish between clear cell and high-grade serous carcinoma of the ovary. Am J Surg Pathol. 2009; 33(1):14-21.

15. Al-Hussaini M, Stockman A, Foster H, McCluggage WG. WT-1 assists in distinguishing ovarian from uterine serous carcinoma and in distinguishing between serous and endometrioid ovarian carcinoma. Histopathology. 2004; 44(2):109-115.

16. Carlson JW, Nucci MR, Brodsky J, Crum CP, Hirsch MS. Biomarker-assisted diagnosis of ovarian, cervical and pulmonary small cell carcinomas: the role of TTF-1, WT-1 and HPV analysis. Histopathology. 2007; 51(3):305-312.

17. Markman M, Petersen J, Belland A, Burg K. CA-125 monitoring in ovarian cancer: patient survey responses to the results of the MRC/EORTC CA-125 Surveillance Trial. Oncology. 2010;78:1-2.

18. Mircea R, Frîncu DL, Dumitrache F. Expression of CA-125 in ovarian cancer. Rev Med Chir Soc Med Nat Iasi. 2009; 113(4):1191-1194.

19. Karaferic A, Jovanovic D, Jelic S. Expression of HER2.neu, estrogen and progesterone receptors, CA-125 and CA19-9 on cancer cell membrane in patients with serous and mucinous carcinoma of the ovary. J BUON 2009; 14(4): 635-639.

20. Wagner N, Michiels JF, Schedl A, Wagner KD. The Milms' tumour suppressor WT1 is involved in endothelial cell proliferation and migration:expression in tumo vessels in vivo. Oncogene 2008; 27: 3662-3672. 